\title{
Management and Sharing of Bibliographies
}

\author{
Erik Wilde, Sai Anand, Petra Zimmermann \\ Swiss Federal Institute of Technology Zürich (ETHZ)
}

\begin{abstract}
Managing bibliographic data is a requirement for many researchers, and in the group setting within which the majority of research takes place, the managing and sharing of bibliographic data is an important facet of organizing the research work. Managing and sharing bibliographies has to balance different levels of shared access (public catalogs, closed research group bibliographies, and personal bibliographies), and the sharing platform should integrate as seamlessly as possible into diverse environments in terms of operating systems, document processing, and other information management tools. The ShaRef system presented in this paper has been designed to fill the gap between public libraries and personal bibliographies, and provides an open platform for sharing bibliographic data among user groups. Through its simple and flexible data model and system architecture, ShaRef adapts to many settings and requirements, and can be used to increase collaboration and information flow within groups.
\end{abstract}

\section{Introduction}

Digital libraries and access technologies have made it very easy for researchers to find relevant information resources. However, once these resources have been found, it is still a challenge to store and manage them in a structured way, and even more so in a group setting with very diverse requirements in terms of operating systems, document processing software, and other information management tools. The Shared References (ShaRef) system presented in this paper provides a solutions to these problems. It implements an open platform which can be adapted to many different research settings. Using ShaRef, research groups can improve the internal information flow, and they can also improve the reuse of information, for example for reading lists for lectures, or for yearly publication lists of a research group.

Section 2 describes the problem of personal information management with regard to bibliographic resources. It shows that sharing and collaborative work are important facets of working with bibliographies, and should be supported by tools designed for managing bibliographies. Sections 3 and 4 describe the ShaRef system, by first describing the data model and some of its implications, and then the system architecture. Sections 5 and 6 discuss integration aspects of ShaRef into existing environments, and example scenarios, thereby describing how the system is able to support the bibliography management requirements of research groups. Section 7 describes some related work and compares the approaches of other system with the design choices made in ShaRef. 


\section{Personal Information Management}

Working with bibliographic references is as close as many scientists will ever get to a formalized and structured way of knowledge management [1]. In addition to the personal management of bibliographic information, sharing of this information about encountered resources also is an important activity, as shown by MARSHALL and BLY [2]. In particular, communicating about the encountered resources and creating a repository which can be used by all members of a group, is an activity taking place in many groups. If the tools do not explicitly support this type of activity, group members will use other tools for performing this task, such as emailing reference or snippets from resources.

In a study conducted by STEINEROvá [3], it is shown that the majority of users underestimate the efforts of seeking information about resources, and that the role of collaboration in the information seeking process often is not recognized properly. The study also shows that many researchers prefer to work alone when seeking information, and that there is a need to provide them collaborative tools, train them for using these tools properly, and establish a culture of information seeking which focuses more on collaborative work rather than individual efforts.

Merging personal information management with collaboration features means that the role of annotations becomes more important, because annotations made by one user can be helpful to other users, and a collaborative system supporting this kind of information exchange enables users to share information in an easily understandable and useful way. Conceptually, annotations can be attached to information resources themselves (i.e., to resources in a digital library), or to the metadata referencing these resources (i.e., to bibliographic references). Agosti et al. [4] propose a comprehensive conceptual model for annotations, which covers digital libraries as well as shared bibliographies (called collaboratories in their paper). The model presented is designed for supporting strong collaboration, a highly structured way of defining the collaboration process. The ShaRef system, on the other hand, is designed to support weak collaboration, a more informal way of collaborating, where the exact workflow of collaboration is left open, but the data model is sufficiently rich to capture the data necessary for sharing information.

\section{Core Design}

The focus of ShaRef is the collaborative management of reference information. The two most important aspects about ShaRef are its data model, described in Section 3.1, and the provided functionality, described in Section 3.2.

\subsection{Data Model}

ShaRef's data model has been designed with the end user in mind, that is it should primarily focus on end users rather than library needs. One key part in the data model is the question of how to model bibliographic references, and in addition to that individual and collaborative features have to be covered such as annotations, group management, and access rights. Because ShaRef focuses on 


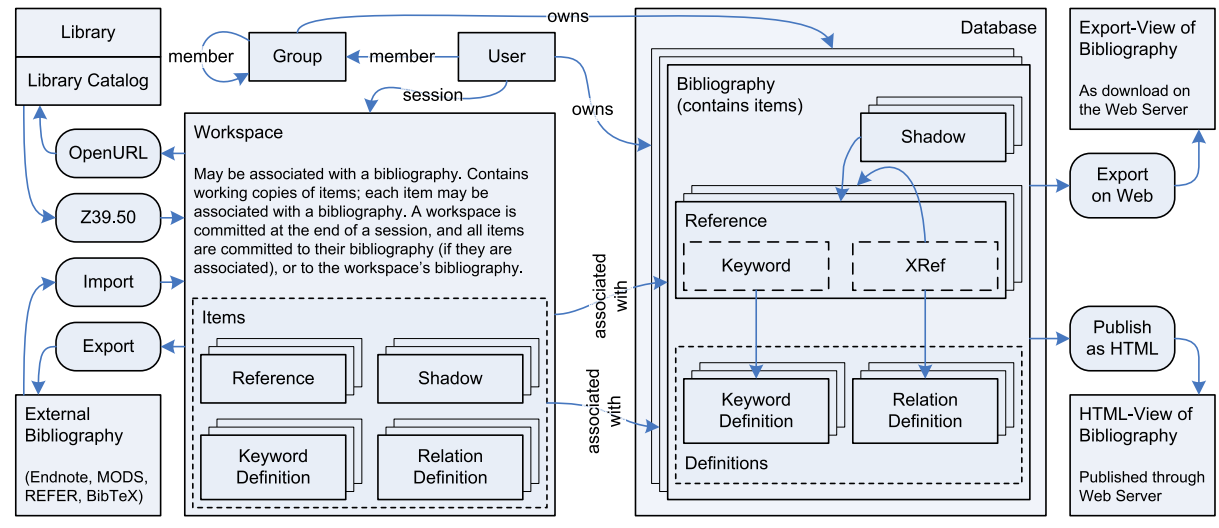

Fig. 1. ShaRef Data Model

end users, complex metadata schemes such as MARC or derived formats have been considered too complex to be useful for end users. On the other hand, specific formats such as $\mathrm{BIBT}_{\mathrm{E}} \mathrm{X}$ or EndNote are also too restrictive because they make it hard to impossible to share data between users coming from different systems. As a consequence, ShaRef's data model has been designed to lie in the middle ground between a union and an intersection between all data formats considered, and mapping rules have been defined which say how to map individual formats to ShaRef's data model ${ }^{1}$. These mapping tables are used for importing and exporting data, and thus define how ShaRef's data model relates to other data models.

The data model for bibliographic references is defined as an XML Schema, which makes it easy to import and export data because of the wide adoption of XML. This schema defines bibliographies to be collections of references, shadows, and keyword and relation definitions, as shown in Figure 1. A reference is the usual set of metadata associated with information resources, designed as the middle ground between a union and an intersection of the most popular formats as described in the paragraph above.

A shadow is a reference to a reference, thus, it is a way how users can mirror other references without actually copying them (this is important for keeping references consistent). The use case for shadows are scenarios where references from a bibliography should be reused in another context, but should still be updated consistently with the original reference. Shadows play an important role in many real-life examples, which are described in Section 6.

Keyword and Relation definitions define concepts which can be used for characterizing references (using keywords to describe it) or dependencies between resources (for example the fact that two resources are identical, or that one resource is a newer version of another resource). The definitions are part of a

\footnotetext{
${ }^{1}$ Available online at http://dret.net/bibconvert/map.
} 
bibliography and can be used for describing references. Keywords and relations can be used in rich text fields, which enable users to create hypertext fragments $^{2}$ describing references. ShaRef makes no attempt at providing semantics or sophisticated support for keywords or relations, thus advanced keyword and keyword hierarchy visualization such as provided by the Technical Report Visualizer $(T R V)$ [5], or complex interpretation of relations such as provided by ClaiMaker [6], are outside the scope of ShaRef. However, ShaRef's data model can handle this information, and through its system architecture (as described in Section 4), it would be possible to plug-in this kind of advanced information visualization as a GUI-level plug-in.

Bibliographies are owned by users or groups, and the group concept is simple. A group consists of users and/or groups, and a subset of group members have adminstration rights and may change the set of group members. For group bibliographies, all group members may write to the bibliography, whereas for personal bibliographies, only the owner may write it and other users may have read access.

The Workspace is the key concept of how ShaRef works. Users never directly interact with a bibliography, they always use a workspace, which can be thought of as a cache or a shopping cart. A workspace contains copies of bibliography items, and may even contain items from multiple bibliographies. During a ShaRef session, users can use any number of workspaces, and each of these workspaces may be associated with a bibliography. User actions changing information only affect the workspace, and if the changes should be made permanent, it has to be committed at the end of a session.

\section{$3.2 \quad$ Functionality}

The data model shown in Figure 1 is the foundation of the ShaRef system. It also is a complete (but rather abstract) depiction of the database schema. Working with this data model through the ShaRef system can be categorized into three different areas, covering the individual tasks to be carried out, collaborative tasks, and administrative functionality. This categorization is also important because the ShaRef system can be used in an online client/server-setting, where collaboration can be supported by the distributed nature of the application, or it can be used in an offline client-only setting, where collaboration is impossible and only individual tasks can be carried out (more about these settings in Section 4 about the system architecture).

- Individual Work: Apart from the usual create, edit, and remove functions provided for workspace items, users can search in bibliographies and get the results as workspace contents, which is the usual way how a workspace is populated initially. One specialty of ShaRef are shadows, which can be used to create virtual bibliographies. For example, when creating a bibliography

\footnotetext{
${ }^{2}$ These fragments are annotations to references and may link to other references (through relations), keywords, or Web resources (using regular URIs).
} 
containing only shadows (i.e., entries pointing to references in other bibliographies), this bibliography will always be up to date when the original references are updated, or it can be frozen at any time by instantiating all shadows (which replaces the shadow with a reference which is a copy of the original reference).

Other important activities are import and export of references, which is described in detail in Section 5. Exporting references typically is used to reuse references, for example as a bibliography for writing a document. Importing enables users to keep their existing references. To make consolidation of references easier when importing references or merging bibliographies, finding duplicates is supported by the system. Catalog access through Z39.50 is also supported and conceptually is not much different from import, because it also retrieves references from an external source and copies them to a workspace. For accessing a catalog from within ShaRef, OpenURLs can be used which make it easy to find catalog entries for ShaRef references.

To better support collaboration, the system supports messaging (as suggested by BRUsh et al. [7]). Messages are sent by other users or generated by the system for certain events (for example, if a periodic link check detected invalid Web links), and they can either be read through the system, or forwarded to an email account to better integrate them into the work environment.

- Collaborative Work: Collaboration in many cases is achieved by simply setting the appropriate access rights on bibliographies, and by assigning bibliographies to the appropriate owners. Group bibliographies can be written by all group members, and personal bibliographies can be written by the owner only. Depending on the required settings (described in more detail in Section 6), the combination of groups, group administrators (who have special privileges for managing the group and its bibliographies), and bibliography configurations provides support for a variety of use cases. ShaRef uses a lightweight group concept, anybody may create groups, and creating new groups and specifying the group's administrators is a simple process. Apart from configuring a bibliography so that it may be read by other users, bibliographies can also be published on the Web (as HTML for providing a human-readable representation, and in predefined export formats for making bibliographies available for download in machine-processable representations). This can be used for providing Web-based access to bibliographies, and it also is a convenient way for accessing a bibliography as long as the access is read only and no sophisticated functionality is required.

- Administrative Tasks: The ShaRef system focuses on the end user, but it uses a client/server architecture, and it may be necessary to perform administrative tasks on the server side. For user identification and authentication, we use a university-wide account system, which helps us to avoid the implementation of user registration, identification, and authentication. Group management is done entirely within ShaRef, because groups play a central role in ShaRef's collaboration model and do not map well to abstractions provided by external user identification and authentication methods. 


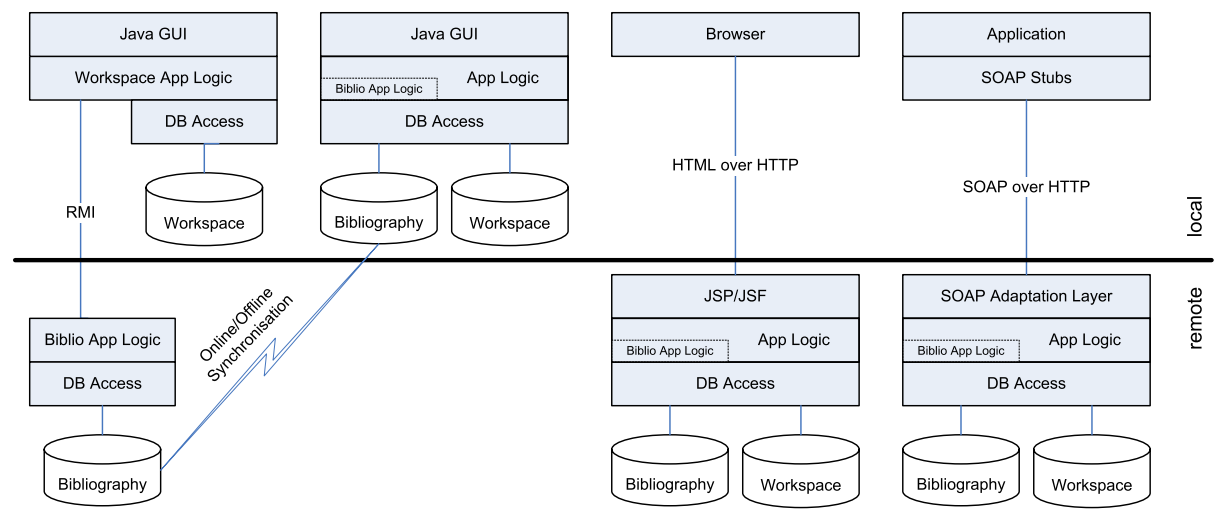

Fig. 2. ShaRef System Architecture

This brief overview of ShaRef's functionality describes the most important features of the system. The system has been kept small because it should be easy to learn, easy to master, and easy to integrate into the work habits of researches from different disciplines. Our goal is to satisfy the needs of most researchers in a group setting, there will be cases where a more complex functionality is required, and in these cases the ShaRef system will not be sufficient. However, ShaRef's design on the data and on the system level makes it easy to extend the system and integrate it into other work environments, which makes it a good candidate for a base system to be extended for the requirements of advanced user groups.

\section{System Architecture}

The system architecture is shown in Figure 2. ShaRef is a Java-based system, because of the platform-independence of Java programs. The system architecture has been designed to support different usage scenarios, with the goal to make the system as flexible as possible. In the user survey that spawned the ShaRef project [8], the majority of users said that offline access was important for managing bibliographies, and thus it was clear that the system needed an online as well as an offline mode.

The overall design of the system is described in more detail in [9]. The data model is exposed as XML, which makes it easy to reuse software components in XML-oriented environments. The online rich client and the offline mode are described in the following sections, they are the most important of ShaRef's usage scenarios. Apart from these two modes, ShaRef also provides a Web-based interface (often called a thin client), and a Web Service API, which makes it possible to integrate ShaRef on the API-level into other applications. Both of 


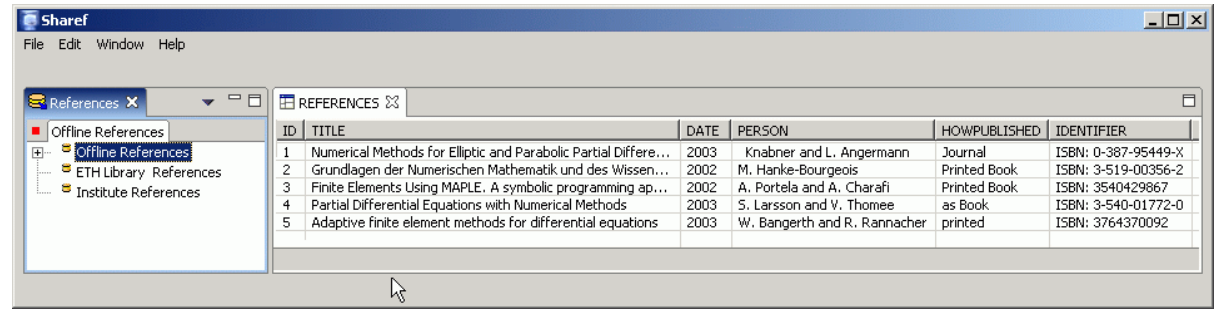

Fig. 3. Table View in the ShaRef Rich Client

these interfaces (thin client and Web Service) do not provide the full functionality available though the Java GUI.

\subsection{Online Mode}

In most cases, ShaRef is used as an online client with a Java GUI, where parts of the application logic are located within the client. For example, workspace management is entirely local, as long as no references are retrieved from or committed to a bibliography. This has been achieved by integrating a pure Java DBMS into ShaRef, which does not compromise ShaRef's portability, and provides good performance figures for handling a couple of thousands of references. Workspaces are represented through a table-oriented representation as shown in Figure 3.

In online mode, client/server communications are implemented using Java RMI, which is ideally suited for our scenario, because the remote interfaces in the online mode have to be reused as local interfaces in offline mode. One of the functions available in online mode is to take bibliographies offline, which creates a copy of the bibliography on the local machine. After taking bibliographies offline, online access is no longer required, so the offline mode is ideally suited for situations where no access to the server is possible, for example on business trips.

\subsection{Offline Mode}

In offline mode, all data is stored locally, and the bibliographies as well as the workspaces are handled by the Java DBMS. Group bibliographies can only be used for reading in the offline mode, while personal bibliographies can also be edited while being offline. In offline mode, the client keeps track of any changes made to the bibliography, and when going online again, a synchronization function updates the online bibliography with the modifications made in offline mode.

\subsection{Web Access}

The online and offline modes described in the previous sections are based on the assumption that the ShaRef client is installed on the local system. This requires 
a Java runtime environment and sufficient access rights to install and run the client code, which may not always be the case. The thin client is an alternative which on the client side only requires a Web browser, but due to the limitations of Web interfaces offers a more limited functionality than the Java client.

Some people may not even be interested in managing their bibliographies with ShaRef, but they would like to use ShaRef's import/export (and thus conversion) capabilities to convert their bibliographic data between different formats. For these people, we have created the bibconvert service (available at http://dret.net/bibconvert/), which only provides the conversion features implemented by the ShaRef project through an easy-to-use Web interface. This way, users can convert their bibliographies without the need to install any code.

\section{Openness}

One of the main goals of ShaRef is to avoid lock-in and to keep the platform as open as possible. In the user study before the project start [8], one of the most frequent comments was that it was unacceptable if a system for bibliography management was designed in a way which made it hard or impossible to import and export data. Many researchers tend to keep their bibliographies over the course of several years, often throughout the whole career. Thus, a system forcing onto them a model into which they cannot import their existing data, and from which they cannot detach when they want to do so, would not be acceptable.

In our survey, the vast majority of users maintaining bibliographies used either EndNote $(55 \%)$ or BiBTEX $(28 \%)$, the rest being mostly database solutions or rather exotic approaches such as spreadsheets. Thus, importing from EndNote and BIBTEX is very important and should be possible without losing any significant information. As it turned out, EndNote's as well as BIBTEX's data models are rather arcane and sometimes do not match very well, which is the reason why we defined a new data model (Section 3.1). However, importing and exporting EndNote and BIBTEX has been designed with a lot of effort, so that the mapping to and from the ShaRef data model is almost lossless (apart from some rather intricate features of the two data models).

In addition to the popular EndNote and BiBTEX formats, ShaRef also supports a number of other formats, which are shown in Table 1. The table lists the import and export support, and naturally some formats are only supported in one direction, such as HTML export. The table also lists the supported formats for a number of other tools and/or services, which have been selected as supporting various bibliography formats.

Apart from the supported formats shown in Table 1, some tools or services also support more specific formats, such as the Pubmed and ISI Web of Science import formats supported by bibutils. However, it should also be noted that only very few of the import and export conversions are lossless, many conversions cannot be lossless because of principal incompatibilities of the data models, and many tools and services do not implement import and export in the best possible way for the given conversion. 


\begin{tabular}{|r||c|c|c|c|c|c|c|}
\hline & BiвTEX & EndNote & FrameMaker & HTML & MODS & REFER & $\begin{array}{l}\text { Reference } \\
\text { Manager }\end{array}$ \\
\hline \hline bibutils & $\checkmark / \checkmark$ & & & & $\checkmark / \checkmark$ & $\checkmark / \checkmark$ & $\checkmark / \checkmark$ (RIS) \\
\hline EndNote & & $\checkmark / \checkmark$ & & $-/ \checkmark$ & & $\checkmark / \checkmark$ & $\checkmark /-$ (RIS) \\
\hline InterBib & $\checkmark / \checkmark$ & & $-/ \checkmark($ MIF) & $-/ \checkmark$ & & $\checkmark / \checkmark$ & \\
\hline $\begin{array}{r}\text { Open Citation } \\
\text { Converters }\end{array}$ & $\checkmark / \checkmark$ & & & & & $\checkmark / \checkmark$ & \\
\hline ShaRef & $\checkmark / \checkmark$ & $\checkmark / \checkmark(\mathrm{XML})$ & & $-/ \checkmark$ & $\checkmark / \checkmark$ & $\checkmark / \checkmark$ & \\
\hline
\end{tabular}

Table 1. Import/Export Support of Bibliography Conversion Tools/Services

\subsection{Import}

Importing references is important because it enables users to start with their existing bibliography, rather than having to start from scratch. Ideally, importing in a group should also work when group members have been using different tools, and are moving to ShaRef for collaboration purposes. However, the principal differences between the legacy data models can make seamless import and integration hard to impossible. For example, in $\mathrm{BIBT}_{\mathrm{EX}}$ each reference has a key, which is defined by the user and used in the Icite command to use this reference in a $\mathrm{HAT}_{\mathrm{E}} \mathrm{X}$ document. In EndNote, however, there is no such key, because the integration of EndNote and MS Word has been solved without requiring a key to exist. Thus, when importing EndNote, no key is present, and therefore no key can used for using this reference with BIBTEX. This problem has been solved by generating keys, but illustrates the problem of merging different data models into a new, collaborative environment.

Import not only enables users to keep their bibliographic data when switching to ShaRef, it also enables new uses for the bibliographic data. For example, when importing data into ShaRef, catalog access through OpenURL is supported through the ShaRef Java client and through HTML representations of the data. By using this feature, users can easily find the resources their references point to, and when they move to another institution with a different library system, they simply have to update ShaRef's OpenURL configuration and can start searching and locating resources in the new library system.

\subsection{Export}

ShaRef is designed to support sharing of references, but is not designed as a tool for directly aiding in document preparation. When users want to use their references for document preparation, they have to export the data to a format supported by their document preparation system, most likely EndNote for MS Word and $\mathrm{BIBT}_{\mathrm{E} X}$ for $\mathrm{LAT}_{\mathrm{E}} \mathrm{X}$. This is not optimal in terms of tool integration, and other systems (e.g., BibShare [10]) have made greater efforts to provide a 
seamless integration into the document preparation system. On the contrary, we decided to concentrate our efforts on the management and sharing facets, and avoid the complexities of directly integrating the tool into a document preparation system.

Another obvious export scenario is the publication of HTML pages, which on the one hand is implemented in the Web publishing features and the Web access described in Section 4.3, but also can be used as an export format, in which case the user gets a set of HTML pages and can use these for Web publication of bibliographic data totally independent from the ShaRef system.

As a last example of data export, we added an export filter to generate XML data which can be directly imported into the Silva content management system (CMS), which is the university-wide system for managing Web pages. This way, research groups can update their publication list on their Web site with one single export operation, whereas before it was necessary to manually enter new publications in the CMS, which not only was a lot of effort, but also led to a wide variety of different publication list styles on different Web sites of the university. With ShaRef's CMS export feature, maintaining publication lists has become easier, and all publication lists use a consistent design.

\section{Example Scenarios}

The data model and system architecture of ShaRef has been designed to provide a wide variety of usage scenarios. This design has been result of the initial study before the project start [8], which showed that there are many different ways of how researchers and research groups use bibliographic data. The following list is an illustration of the range of possible usage scenarios:

- Group Publication Databases: A research group is defined as a group within ShaRef, and then a group bibliography is created which can be written and modified by all group members. If a more controlled bibliography is required, a new group is created with the designated bibliography administrators. The bibliography is assigned to this new group, while the research group is granted read-only access. In both cases, group members can still maintain their personal bibliographies and simply create shadows of their personal bibliography. For using the publication database in different applications, and maybe for making it accessible on the Web and for download, the export features described in Section 5.2 can be used.

- Controlled Vocabularies: In many application areas, keywords to be used for classifying references should be taken from a controlled vocabulary. This can be achieved by creating a bibliography containing only keyword definitions (the controlled vocabulary), and then using the keywords from this bibliography in other bibliographies. Depending on who should be authorized to make changes to the controlled vocabulary, the bibliography containing the keyword definitions should be set up as a personal or group bibliography. Through the import feature of ShaRef, it is also possible to import an existing vocabulary, by transforming it to ShaRef XML and then import it into the (previously empty) bibliography. 
- Reading Lists: In academic settings, reading lists (for lectures or seminars) are frequently required. In order to make these reading lists as easily accessible as possible. By creating a new bibliography for each reading lists, and only using shadows within this bibliography, the reading list can be created as a view of a part of the underlying bibliography. Through ShaRef's OpenURL functionality, students will even be able to go from the reading list to the university's library catalog with a single click.

These usage scenarios give a brief overview of how ShaRef's relatively simple model of bibliographies, users, and groups can be used to implement many of the typical usages of bibliographic data.

\section{Related Work}

When comparing work on personal bibliography management tools with work on improving access technologies to digital libraries, it can be seen that surprisingly little effort goes into this area of research. However, apart from personalization features of library access systems, there are also some systems which are more specifically geared towards personal tools.

The Kepler system [11] chooses a different approach than ShaRef, because it focuses on making the personal bibliographies available as publicly accessible catalogs through OAI-PMH. Kepler enables users to collaborate through aggregating data in a Group Digital Library (GDL). Media Matrix [12] enables users to create secondary repositories, which means that users can reuse digital media found on the Web and in repositories, and can create their own repository of them. The main focus of media matrix are multimedia contents, and it provides many features for handling different media types. CDS [13] and DSpace [14] are other examples for systems with collaboration features, and Reference Manager is a commercial product offering collaboration features.

In the information management field, there is a trend towards institutional repositories [15] at different (and maybe multiple) levels, and even though there is ongoing work and some systems are used already, this field of research is still very active.

\section{Conclusions}

While the Java Client is still in its prototype stage, the bibconvert service is publicly available and is used for converting bibliographies from one format to another, and for converting bibliographies to the XML format supported by our university's CMS. The feedback so far has been very positive, because many researchers experience the management of bibliographic data as a process not well supported by many of today's tools. During the summer of 2005, we will give introductory courses for university employees and students. The projects ends in December 2005, and we expect that ShaRef's unique features as an open and collaborative system for managing bibliographic data will establish it as a useful tool for many researchers and students. 


\section{References}

1. Wilde, E.: References as Knowledge Management. Issues in Science \& Technology Librarianship (2004)

2. Marshall, C.C., Bly, S.A.: Sharing Encountered Information: Digital Libraries get a Social Life. In Chen, H., Wactlar, H.D., chih Chen, C., Lim, E.P., Christel, M.G., eds.: Proceedings of the Fourth ACM/IEEE Joint Conference on Digital Libraries, Tucson, Arizona, ACM Press (2004) 218-227

3. Steinerová, J.: In Search for Patterns of User Interaction for Digital Libraries. In Koch, T., Sølvberg, I.T., eds.: Research and Advanced Technology for Digital Libraries: Proceedings of the 7th European Conference on Digital Libraries. Volume 2769 of Lecture Notes in Computer Science., Trondheim, Norway, Springer-Verlag (2003) 13-23

4. Agosti, M., Ferro, N., Frommholz, I., Thiel, U.: Annotations in Digital Libraries and Collaboratories - Facets, Models and Usage. [16] 244-255

5. Ginsburg, M.: Visualizing Digital Libraries with Open Standards. Communications of the Association for Information Systems 13 (2004) 336-356

6. Uren, V., Buckingham Shum, S., Li, G., Domingue, J., Motta, E.: Scholarly Publishing and Argument in Hyperspace. In: Proceedings of the Twelfth International World Wide Web Conference, Budapest, Hungary, ACM Press (2003) 244-250

7. Brush, A.J.B., Bargeron, D., Grudin, J., Gupta, A.: Notification for Shared Annotation of Digital Documents. In: Proceedings of the SIGCHI Conference on Human Factors and Computing Systems, Minneapolis, Minnesota, ACM Press (2002) 8996

8. Wilde, E.: Usage and Management of Collections of References. Technical Report TIK-Report No. 194, Computer Engineering and Networks Laboratory, Swiss Federal Institute of Technology, Zürich, Switzerland (2004)

9. Wilde, E., Anand, S., Zimmermann, P.: ShaRef: XML-Centric Software Design. Technical Report TIK-Report No. 213, Computer Engineering and Networks Laboratory, Swiss Federal Institute of Technology, Zürich, Switzerland (2005)

10. Canós, J.H., Mena, E.: BibShare: An Interoperable System to Access and Maintain Bibliographic References. In: III Jornadas de Trabajo DOLMEN, Madrid, Spain (2002)

11. Maly, K., Nelson, M., Zubair, M., Amrou, A., Kothamasa, S., Wang, L., Luce, R.: Enhancing Kepler Usability and Performance. [16] 317-328

12. Kornbluh, M., Fegan, M., Rehberger, D.: Media Matrix: Creating Secondary Repositories. [16] 329-340

13. Keller Gold, A., Baker, K.S., LeMeur, J.Y., Baldridge, K.: Building FLOW: Federating Libraries on the Web. In Marchionini, G., ed.: Proceedings of the Second ACM/IEEE-CS Joint Conference on Digital Libraries, Portland, Oregon, ACM Press (2002) 287-288

14. Smith, M., Barton, M., Bass, M., Branschofsky, M., McClellan, G., Stuve, D., Tansley, R., Walker, J.H.: DSpace - An Open Source Dynamic Digital Repository. D-Lib Magazine 9 (2003)

15. Crow, R.: The Case for Institutional Repositories: A SPARC Position Paper. Technical report, The Scholarly Publishing \& Academic Resources Coalition, Washington, D.C. (2002)

16. Heery, R., Lyon, L., eds.: Research and Advanced Technology for Digital Libraries: Proceedings of the 8th European Conference on Digital Libraries. In Heery, R., Lyon, L., eds.: Research and Advanced Technology for Digital Libraries: Proceedings of the 8th European Conference on Digital Libraries. Volume 3232 of Lecture Notes in Computer Science., Bath, UK, Springer-Verlag (2004) 